\title{
DISTURBANCES IN NIGHT VISION AFTER EXCIMER LASER PHOTOREFRACTIVE KERATECTOMY
}

\author{
DAVID P. S. O'BRART ${ }^{1}$, CHRIS P. LOHMANN ${ }^{1,3}$, FRED W. FITZKE $^{2}$, GREGORY KLONOS ${ }^{1}$, \\ MELANIE C. CORBETT ${ }^{1}$, MALCOLM G. KERR-MUIR ${ }^{1}$ and JOHN MARSHALL ${ }^{1}$ \\ London and Regensberg, Germany
}

\begin{abstract}
SUMMARY
Eighty-four patients with up to $-\mathbf{6 . 0 0}$ dioptres of myopia underwent photorefractive keratectomy (PRK), using $5.00 \mathrm{~mm}$ ablation zones. Three months post-operatively $38(45 \%)$ complained of disturbances in night vision, compared with $21(25 \%)$ pre-operatively. In the majority, these disturbances were regarded as negligible. However, $9(\mathbf{1 1 \%})$ reported significant problems, defined as an inability to drive safely at night with the treated eye. At 12 months, 32 patients $(38 \%)$ complained of impaired night vision, $4(5 \%)$ of whom had significant problems. A series of measurements were performed to investigate the origins of these disturbances, especially in patients reporting significant problems. Visual impairment from forward scattered light was investigated using a computerised technique. Back scattered light was measured with a charge coupled device-camera system and a computer program was used to assess the degree of halation around a bright light source on a high-resolution monitor. Pupillary diameters were measured by infrared television pupillometry. At 6 months, those reporting a starburst effect around lights at night had small hyperopic shifts, minimal halos and high forward and back light scatter measurements. Patients who reported halo phenomena had large hyperopic shifts, little light scatter and large pupillary diameters. Of the 4 patients who reported significant disturbances at 12 months, all had persistent halo problems. Those with starburst effects in the early postoperative period noticed an improvement with time as their corneal haze gradually improved. Perturbations of night vision after PRK manifest as starbursts and halos around lights. Disturbances in corneal transparency appear to be responsible for starburst effects and are usually transient. Halos are myopic blur circles and may be persistent in a small number of individuals. All
\end{abstract}

From: 'Department of Ophthalmology, St Thomas' Hospital, London, UK; ${ }^{2}$ Institute of Ophthalmology, Bath Street, London, UK; ${ }^{3}$ University Eye Clinic, Regensberg, Germany.

Correspondence to: Mr. D. P. S. O'Brart, FRCS, FRCOphth, Department of Ophthalmology, St Thomas' Hospital, Lambeth Palace Road, London SE1 7EH, UK. patients should be informed pre-operatively of the possible consequences of disturbances in night vision.

Excimer laser photorefractive keratectomy (PRK) has been the subject of clinical trials for over 3 years and to date over 70000 procedures have been performed worldwide. This number is due to rise rapidly over the next few years with the inexorable increase in the number of excimer laser systems in use. It appears to be a safe and effective procedure for the correction of low degrees of myopia ${ }^{1-4}$ and research continues into the treatment of other refractive disorders. ${ }^{5.6}$ It is the first widely available form of refractive surgery performed directly on the optical zone of the cornea and thus concern has been raised about the possibility of disturbances in visual performance post-operatively.

Despite a transient loss of corneal transparency, few patients report daytime disturbances in vision and best corrected Snellen visual acuity typically returns to preoperative levels within a few months after treatment. ${ }^{1-4}$ However, disturbances in night vision are often reported and are manifest to patients as 'starbursts' and 'halos' around bright point sources of light.

Gartry et al. ${ }^{1}$ reported a series of 120 sighted eyes treated with a maximum ablation diameter of $4.00 \mathrm{~mm}$. On direct questioning 94 patients $(78 \%)$ reported shimmering rings around lights at night, which were worse in the early post-operative period. Twelve patients $(10 \%)$ considered that these disturbances were sufficient to interfere with night driving and declined treatment of their second eye.

In order to introduce a degree of objectivity into the assessment of reduced night vision after PRK we have designed two computer programs. The first measures the effect of light scatter from disturbances in corneal transparency. ${ }^{7}$ The second measures the magnitude of halation around a bright light source generated on a high-resolution monitor. ${ }^{8}$ Using the halo program, night halo after PRK has been shown to be diminished by using either artificial pupils or negative lens overcorrection. In individuals with 
identical bilateral corrections, except for ablation zone size, there were found to be fewer halos with $5.00 \mathrm{~mm}$ than with $4.00 \mathrm{~mm}$ zones. ${ }^{8}$ These observations confirm the concept that the halo effect is a myopic blur circle produced by the refraction of light by the untreated paracentral cornea. The magnitude of the blur circle is dependent upon the ratio of pupil size and the diameter of the ablation zone. It is therefore only apparent in mesopic/scotopic conditions.

Night halo phenomena are not unique to patients who have undergone PRK. They have been observed in those myopes corrected with contact lenses, particularly hard lenses, where they are thought to arise from a mismatch in curvature between the lens and peripheral cornea. ${ }^{9}$

There appears to be a wide individual variation in the degree of disability generated by disturbances in night vision after PRK. In order to establish the time base and origins of such problems, we documented the perturbations in night vision reported by a series of 84 patients treated with $5.00 \mathrm{~mm}$ ablation zones over a 12 month follow-up period. Measurements of disturbances in corneal transparency and light scatter ${ }^{7.10 .11}$ and halo ${ }^{8}$ were made in these patients. This allowed patients to be divided into those whose night vision problems were predominantly generated by corneal haze and those who had disturbances caused by halos. On the basis of these data, strategies to prevent, reduce and manage disturbances of night vision after PRK are presented.

\section{PATIENTS AND METHODS}

\section{Patients}

There were 84 patients ( 84 eyes) who underwent PRK for correction of myopia. The average age was 36 years, with a range of 24-68 years. There were 44 patients who had -3.00 dioptres (D) and 40 with -6.00 D corrections, with each patient being corrected to the nearest dioptre. The mean pre-operative refraction (spherical equivalent) was $-4.5 \mathrm{D}$, range $-2.5 \mathrm{D}$ to $-7.00 \mathrm{D}$.

\section{Patient Assessment and Post-operative Management}

All patients were counselled fully prior to PRK to discuss the investigative nature of the surgery. Past optometric records were scrutinised to ensure stability of refraction and all subjects were over the age of 24 years. All eyes had less than $1.50 \mathrm{D}$ of astigmatism. Patients with pre-existing ocular pathology, diabetes and connective tissue disorders were excluded.

Pre-operatively a detailed ocular examination was performed and included refraction, keratometry, slit lamp examination, tonometry and mydriatic fundoscopy.

The operative procedure has been described elsewhere.' In all patients, chloramphenicol eyedrops q.d.s. were administered for 2 weeks post-operatively. Given the recent findings in a prospective randomised doublemasked trial of the use of topical corticosteroids after $\mathrm{PRK},{ }^{12}$ the patients were divided into two groups. In one group 30 eyes ( $16-3.00 \mathrm{D}$ and $14-6.00 \mathrm{D}$ corrections) received fluorometholone $0.1 \%$ eyedrops 8 times a day for 1 month, with a reducing dosage over the next 5 months. In the second group of 54 eyes $(28-3.00 \mathrm{D}$ and $26-6.00 \mathrm{D}$ corrections) no topical corticosteroids were administered.

Post-operative examinations were carried out at 3,6,9 and 12 months and included refraction, keratometry, biomicroscopy and tonometry. In addition, measurements of objective haze were made with a charge coupled devicecamera system, the calibration and data analysis of which have been described in detail. ${ }^{10,11}$ In essence, this device measures either reflection and back scattered light generated by disturbances in corneal transparency or back scattered light alone. The degradation of the retinal image generated by the forward scatter of light was assessed using a recently described computerised technique, which measures visual contrast with a central test stimulus in the presence of a bright, annular, flickering light source.? Measurements of the magnitude of the halation around a central bright light source generated on a high-resolution monitor were made, using a previously described computer program. ${ }^{8}$

At each post-operative visit all patients were questioned, using a standardised, predetermined sequence to ascertain the frequency and severity of night vision problems. All patients with significant disturbances were investigated to determine the underlying cause of their problem, with particular reference to discrimination between haze and halo. This group was defined as those who felt that they would not be able to drive safely at night with their treated eye alone. In addition to the above measurements, apparent pupil diameters were examined using a Whittaker Corporation Series 1800 binocular infrared television pupillometer in darkness ${ }^{13}$ and corneal topography was performed in each patient to determine the centration of the ablated zone, using a Computed Anatomy TMS-1 system.

\section{Excimer Laser}

A Summit Technology ExciMed UV200 excimer laser was used, with an emission wavelength of $193 \mathrm{~nm}$, a fixed pulse repetition rate of $10 \mathrm{~Hz}$ and a radiant exposure of $180 \mathrm{~mJ} / \mathrm{cm}^{2}{ }^{2}$ In this system differential ablation is achieved by successive laser pulses being delivered through an expanding iris diaphragm. The maximum diameter of the diaphragm was $5.00 \mathrm{~mm}$ in all eyes.

\section{RESULTS}

The incidence of night vision disturbances after excimer laser PRK in 84 patients treated with $5.00 \mathrm{~mm}$ ablation zones is shown in Table I. The disturbances were maximal at 3 months and declined thereafter. Of the 84 patients, 21 $(25 \%)$ reported some interference with night vision prior to the procedure. These disturbances were associated with both spectacle and contact lens correction.

Twelve months after PRK, 32 patients reported perturbations of night vision and of this group 17 were individuals who had noticed some problems pre-operatively. 
Table I. Night vision disturbances in 84 patients treated with $5.00 \mathrm{~mm}$ ablation zones over a 12 month follow-up period

\begin{tabular}{lccccc}
\hline & Preop. & 3 months & 6 months & 9 months & 12 months \\
\hline $\begin{array}{l}\text { Total no. patients } \\
\begin{array}{l}\text { No. with night vision } \\
\text { disturbances }\end{array}\end{array}$ & $21(25 \%)$ & 84 & 84 & 84 & 84 \\
$\begin{array}{l}\text { No. with significant } \\
\text { disturbance }\end{array}$ & 0 & $43(51 \%)$ & $38(45 \%)$ & $35(42 \%)$ & $32(38 \%)$ \\
\hline
\end{tabular}

Nineteen had undergone $-6.00 \mathrm{D}$ corrections and 13 had had $-3.00 \mathrm{D}$ corrections. There did not appear to be any association between the use of post-operative topical corticosteroids and night vision disturbances. Halo phenomena were reported by the majority, with only 5 of the 32 patients reporting starburst effects. These disturbances were generally regarded as minor and very few patients reported problems unsolicited.

Measurements of haze, back scattered light, forward light scatter, halo, pupil diameters and hyperopic shift in 9 patients who experienced significant impairment of night vision at 6 months are shown in Table II. Of these, 3 reported a starburst effect around lights at night, 5 described the appearance of shimmering halos, and 1 had a combination of starbursts and halos. All these patients felt that they would not be able to drive safely at night with their treated eye alone.

Of the patients listed in Table II, those with starburst phenomena around bright lights at night had profound corneal haze. These eyes had regressed, with small hyperopic shifts, resulting in undercorrection and small halo measurements. Patients with halo problems after PRK had achieved favourable results with large hyperopic shifts, resulting in a correction near emmetropia, and minimal corneal haze. However, they had large pupil diameters in darkness, above the 50th centile for their age group, compared with previous published data. ${ }^{14}$

Corneal topography gave no indication of significant decentration of the ablation zone in any of the 9 problem patients. The centre of the treated zone was within $0.5 \mathrm{~mm}$ of the entrance pupil centre in each case.

In Table II, the results at 12 months for hyperopic shift, haze, back and forward light scatter and halo measurements are shown in parentheses. Those who reported significant disturbances associated with starburst effects in the early post-operative period, noticed an improvement with time as their corneal haze gradually improved. However, there were still 4 patients with significant night vision problems 12 months post-operatively and these individuals are highlighted. All these patients complained of halo phenomena and 2 declined treatment of their second eyes despite good refractive outcomes. Patient 2 noted an improvement in night vision with a reduced halo at 12 months. This was associated with a reduction in her hyperopic shift and the use of a contact lens to treat the induced hypermetropia.

\section{DISCUSSION}

Reduced night vision after PRK appears to have two inde-

Table II. Details of 9 patients who reported significant night vision problems 6 months after PRK with a $5.00 \mathrm{~mm}$ ablation zone (The numbers in parentheses are the values at 12 months)

\begin{tabular}{|c|c|c|c|c|c|c|c|}
\hline \multirow[b]{2}{*}{$\begin{array}{l}\text { Patient } \\
\text { no. }\end{array}$} & \multirow[b]{2}{*}{$\begin{array}{c}\text { Age } \\
\text { (years) }\end{array}$} & \multirow[b]{2}{*}{$\begin{array}{l}\text { Hyperopic shift } \\
\text { (dioptres) }\end{array}$} & \multicolumn{3}{|c|}{ Haze } & \multirow[b]{2}{*}{$\begin{array}{l}\text { Halo } \\
\left(\mathrm{mm}^{2}\right)\end{array}$} & \multirow[b]{2}{*}{$\begin{array}{l}\text { Pupil } \\
(\mathrm{mm})\end{array}$} \\
\hline & & & $\begin{array}{l}\text { Reflected and back } \\
\text { scatter (GSU) }\end{array}$ & $\begin{array}{l}\text { Back scatter } \\
\text { (GSU) }\end{array}$ & $\begin{array}{l}\text { Forward scatter } \\
\text { (\% contrast })\end{array}$ & & \\
\hline \multicolumn{8}{|c|}{ (a) Patients reporting halo phenomena } \\
\hline $1^{\mathrm{a}}$ & 34 & $5.375(4.75)$ & $166(150)$ & $126(115)$ & $15.6(4.4)$ & $4230(2841)$ & 7.85 \\
\hline $2^{b}$ & 42 & $9.825(8.5)$ & $141(160)$ & $113(129)$ & $15.5(11.8)$ & $11653(7712)$ & 7.7 \\
\hline $3^{\mathrm{a}}$ & 26 & $3.25 \quad(3.25)$ & $174(131)$ & $118(106)$ & $8.1(7.0)$ & $1711(1787)$ & 7.25 \\
\hline $4^{\mathrm{a}}$ & 29 & $3.00(2.875)$ & $163(144)$ & $124(108)$ & $10.2(15.5)$ & $2735(1385)$ & 7.3 \\
\hline $5^{\mathrm{a}}$ & 37 & $3.875(3.5)$ & $152(171)$ & $126(132)$ & $9.1 \quad(9.6)$ & 1912 (1912) & 6.75 \\
\hline \multicolumn{8}{|c|}{ (b) Patients reporting starburst phenomena } \\
\hline $6^{\mathrm{c}}$ & 37 & $1.625(1.625)$ & 239 (199) & $214(147)$ & $27 \quad(17.2)$ & $409 \quad(501)$ & 6.4 \\
\hline $7^{\mathrm{c}}$ & 25 & $1.5 \quad(2.75)$ & $244(230)$ & $242(179)$ & $27.3(9.2)$ & $513 \quad(568)$ & 7.0 \\
\hline $8^{c}$ & 34 & $1.0 \quad(1.375)$ & $242(134)$ & $228(109)$ & $24.3(14.3)$ & 1188 & 6.3 \\
\hline \multicolumn{8}{|c|}{ (c) Patient reporting a combination of halos and starbursts } \\
\hline $9^{c}$ & 27 & $5.375(4.0)$ & $242(181)$ & $228(132)$ & $(3.2)$ & 4199 (1209) & 7.7 \\
\hline
\end{tabular}

All measurements of objective haze are in Grey Scale Units (GSU).

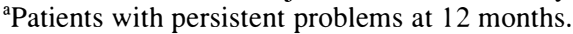

${ }^{b}$ Hyperopic shift reduced 6 months after PRK with an improvement in night halo.

${ }^{c}$ Night vision problems had resolved by 12 months post-operatively with a reduction in haze and light scatter measurements.

Normal pre-operative values:

Reflected and back light scatter: mean $=137 \mathrm{GSU}$ (range 105-168), $n=84$.

Back light scatter: mean $=121$ GSU (range 96-143), $n=84$.

Forward light scatter: mean $=8.6 \%$ contrast $($ range $1.6-19.7), n=100$.

Halo measurement: mean $=435 \mathrm{~mm}^{2}$ (range $\left.0-1344\right), n=100$ myopes with best spectacle correction. 
pendent causal elements. The first is degradation of the retinal image by the forward scatter of light as it passes through corneal haze. The second is the generation of myopic blur circles. Myopic blur circles result from two separate refracting surfaces within the optical zone of the cornea, both of which contribute to the retinal image when the pupil diameter extends beyond the area of the ablated zone.

Our studies indicate that the forward light scatter associated with corneal haze may result in a starburst effect seen around lights at night (Table II). This phenomenon is particularly apparent around bright light sources surrounded by low ambient levels of illumination. A typical example of these conditions is urban street lighting and at night this environment can be particularly disabling. It is difficult to relate the magnitude of disturbances in a patient's vision to subjective clinical observations of haze. ${ }^{10,11}$ Clinical observations consist of a slit lamp image in which the haze is seen as an altered light reflex. This image is generated by two components. The first is light reflected from atypical cellular and extracellular structures in the healing cornea. The second is light scattered back towards the observer from these structures. ${ }^{10.11}$ Neither of these components makes a contribution to the patient's image, which consists entirely of transmitted and forward scattered light. The more forward scatter of light, the greater the degradation of the retinal image. ${ }^{7}$ Haze first becomes apparent a few weeks post-operatively, is maximal at 3-4 months and then gradually fades. ${ }^{10.11}$ The magnitude and timing of starburst phenomena are related to those of corneal haze and in our patients were at their worst 3-6 months after PRK (Table I). As haze decreases, the starburst effect usually reduces to an insignificant level and therefore in most patients causes only transient interference with night vision. Although we identified a number of individuals with disabling starbursts associated with high haze and light scatter measurements in the early post-operative period, few patients showed persistence of these problems 12 months after PRK (Table II).

Most eyes with significant haze also show higher degrees of regression. ${ }^{12}$ The combination of high haze and regression of the intended correction may give rise to complex night vision disturbances. In such patients the problems may be caused partially by forward light scatter and partially by a poorly formed retinal image due to residual myopia.

The forward scatter of light producing starbursts is not unique to PRK and is experienced by many myopes corrected with soft contact lenses. ${ }^{7}$ In PRK the scatter centres are abnormal keratocytes, intrastromal vacuoles and atypical collagen. ${ }^{10}$ In soft contact lenses they may be lens deposits or surface irregularities induced by lens dehydration. ${ }^{7.15}$ Starburst phenomena are also described by some patients after radial keratotomy (RK) ${ }^{16}$ In such cases, starbursts seem to be caused where the scars of the radial incisions extend over the pupil margins. The scar geometry appears to determine the axes of the rays of the starburst. ${ }^{16}$

In addition to starburst phenomena, some patients describe the appearance of shimmering rings around point sources of light at night. Patients may mistakenly describe this phenomenon as 'glare'. These shimmering rings or halos are myopic blur circles and are usually considered insignificant. In a small minority they may cause sufficient disturbance to interfere with night driving. Unlike haze, halo may be a persistent problem. In our series of eyes treated with $5.00 \mathrm{~mm}$ ablation zones, $4(5 \%)$ patients reported significant problems at 12 months. In 120 eyes treated with $4.00 \mathrm{~mm}$ ablation zones reported by Gartry et $a l .{ }^{17}$ there were 12 eyes (10\%) with interference of night driving almost 2 years after PRK.

PRK halos differ from the normal physiological entoptic halos. These have been extensively investigated ${ }^{18}$ and have been described as a series of up to four separate, concentric rings of alternating intensity. They are assumed to be the result of diffraction by normal anatomical structures within the ocular media. They should not be confused with the myopic blur circles, which are confluent with the light source, more apparent and larger than entoptic halos. ${ }^{8}$

Patients become aware of night halos soon after surgery, during the period of initial overcorrection. In most they are considered negligible a few months after surgery, when the initial excess hyperopic shift has reduced. For higher corrections, this time course may be retarded by the use of topical corticosteroids, when eyes may remain overcorrected while on treatment. ${ }^{12}$ It has been suggested that there may be some cortical adaptation producing reduced awareness with time. Claims have also been made that the perception of halo may recede when patients have undergone a procedure on their second eye. Our clinical experience with over 500 PRK procedures has not supported this.

The magnitude of halo after PRK and the resultant degree of impediment perceived by individual patients is very variable. Typically, those patients with large halos have had good corrections with large hyperopic shifts, little corneal haze and minimal light scatter measurements (Table II). However, pupil diameters in these individuals were found to be conspicuously large, when measured by infrared television pupillometry. In most cases they were greater than $7.00 \mathrm{~mm}$ and therefore above the 50th percentile for age. ${ }^{14}$

The variation in disturbance of night vision does not seem to be related to the absolute size of the halo. Some patients in our series with very large halos did not complain of serious problems. Optical considerations would suggest that the ratio of the relative retinal irradiance between the image and the blur circle is of more critical importance than the absolute size of the halo. The higher the irradiance of the latter, the more disabling the effect. These observations are further supported by findings that the magnitudes of halo measurements in myopes corrected with hard contact lenses are much greater than those with PRK. ${ }^{9}$ Although many hard contact lens wearers notice some halo disturbance at night, they do not usually report significant problems. Such halos are 
thought to arise from a gradual mismatch in curvature between the cornea and lens periphery. This would result in less intense blur circles than those experienced after PRK, where there is a sudden increase in dioptric power at the edge of the ablated zone. A systematic investigation of this would require a measurement of visual function within the area of the blur circle.

Lifestyle appears to be important in the perception of halos and in our experience patients who seldom drive at night do not usually consider them a problem. The road conditions at night will also influence the perception of the blur circles. Patients often report that the disability is greater on poorly lit roads and less troublesome with normal urban street-lighting, where the higher frequency of light sources and greater luminance result in smaller pupil sizes. Seasonal variation in daylight may influence the severity of symptoms and patients in which the early post-operative period coincides with the winter months may complain more frequently.

Given that younger individuals have larger pupils, ${ }^{14}$ it is more likely that in this group more disabling halos will occur with small diameter ablations and larger dioptric corrections. Those patients who have pupil diameters greater than $7.00 \mathrm{~mm}$, when measured in darkness (Table II), are more likely to have troublesome halos and should be informed that they may have post-operative problems. Professional drivers and patients who regularly drive at night, especially on motorways and roads without lighting, should be made fully aware of the potential night vision problems after PRK.

The incidence of significant night halo could be reduced further by increasing the ablation diameter. This would, however, result in a greater ablation depth per dioptre ${ }^{19}$ and might increase the propensity for anterior stromal haze. An alternative to increasing the beam diameter might be to modify the algorithm to create a new ablation profile. In this case blended peripheral transition zones would create wider ablations while maintaining shallow ablation depths. With the move towards larger beam diameters, halos should become less of a problem in future studies. Attempts to cope with problem patients generated by $4.00 \mathrm{~mm}$ PRK exposures have only been marginally successful.

We have shown previously that the halo after PRK was reduced with negative lenses or pinholes. ${ }^{8}$ In some individuals with persistent problems it has been helpful to prescribe weak negative lens overcorrection for night driving. Weak miotics instilled 30 minutes before travelling at night have also helped, although the long-term use of miotics in young individuals is an unsatisfactory answer to this problem. In a small group of $4.00 \mathrm{~mm}$ PRK patients reablation has been carried out using $5.00 \mathrm{~mm}$ and $6.00 \mathrm{~mm}$ ablation diameters. However, this is only suitable for individuals with residual myopia.

Myopes often suffer some degradation of night vision regardless of the type of correction for their refractive error. Although the majority of PRK patients are satisfied with the outcome of their procedure, all patients must be informed pre-operatively of the possible consequences for their night vision of haze and halos. It should be emphasised that haze will cause only a transitory disturbance in night vision with normal performance 6-9 months after surgery, whilst halos if present may be a persistent problem.

The authors acknowledge the Iris Fund for Prevention of Blindness for continued financial support in relation to both the purchase and maintenance of the laser and provision of a Research Fellowship for the principal investigator, David O'Brart. Miss Corbett holds the William's Fellowship for Medical and Scientific Research of the University of London. Professor Marshall is a consultant for Summit Technology.

The authors thank Professor S. E. Smith, Mrs. Ann Patmore, Sister Anne Welch and Mrs. Judith Armstrong for clinical assistance and technical support. Finally, they thank Mrs. June Kraft for her secretarial assistance.

\section{REFERENCES}

1. Gartry DS, Kerr Muir MG, Marshall J. Photorefractive keratectomy with an argon fluoride excimer laser: a clinical study. Refract Corneal Surg 1991;7:420-35.

2. Seiler T, Wollensak J. Myopic photorefractive keratectomy with the excimer laser. Ophthalmology 1991;98:1156-63.

3. McDonald MB, Liu JC, Byrd TJ, Abelmegeed M, Andrade HA, Klyce SD, et al. Central photorefractive keratectomy for myopia. Ophthalmology 1991;98:1327-37.

4. Sher NA, Chen V, Bowers RA, Frantz JM, Brown DC, Eiferman $\mathrm{R}$, et al. The use of the excimer laser for myopic photorefractive keratectomy in sighted eyes. Arch Ophthalmol 1991;109:1525-30.

5. McDonnell PJ, Moreira H, Garbus J, Clapham TN, D'Arcy J, Munnerlyn CR. Photorefractive keratectomy for astigmatism: initial clinical results. Arch Ophthalmol 1991; 109:1370-3.

6. Gordon M, Brint SF, Durrie DS, Seiler T, Friedman MD, Johnson NMF, et al. Photorefractive keratectomy (PRK) at $193 \mathrm{~nm}$ using an erodible mask. SPIE Proc 1992;1644.

7. Lohmann CP, Fizke FW, O'Brart DPS, Kerr Muir MG, Timberlake G, Marshall J. Corneal light scattering and visual performance in myopes: a comparison between spectacles, contact lenses and excimer laser photorefractive keratectomy. Am J Ophthalmol 1993;115:444-53.

8. O'Brart DPS, Lohmann CP, Fitzke FW, Smith SE, Kerr Muir MG, Marshall J. Night vision disturbances after excimer laser photorefractive keratectomy: haze and halos. Eur J Ophthalmol 1993;4:159-67.

9. Lohmann CP, Fitzke FW, O'Brart DPS, Kerr Muir MG, Marshall J. Halos: a problem for all myopes? A comparison between spectacles, contact lenses and photorefractive keratectomy. Refract Corneal Surg (Suppl) 1993;9:S72-5.

10. Lohmann CP, Gartry D, Kerr Muir M, Timberlake G, Fitzke F, Marshall J. Haze in photorefractive keratectomy: its origins and consequences. Laser Light Ophthalmol 1991; 4:15-34.

11. Lohmann CP, Gartry DS, Kerr Muir M, Timberlake GT, Fitzke FW, Marshall J. Corneal haze after excimer laser refractive surgery: objective measurements and functional implications. Eur J Ophthalmol 1991;1:173-80.

12. Gartry DS, Kerr Muir MG, Lohmann CP, Marshall J. The effect of topical corticosteroids on refractive outcome and corneal haze after photorefractive keratectomy. Arch Ophthalmol 1992;110:944-52.

13. Lowenstein O, Loewenfeld IE. Electronic pupillography: a new instrument and some clinical applications. Arch Ophthalmol 1958;59:352-62.

14. Smith SA. Pupillary function in autonomic failure. In: Ban- 
nister R, editor. Autonomic failure. 2nd ed. Oxford: Oxford University Press, 1988:401.

15. Timberlake GT, Doane MG, Bertra JH. Short-term, lowcontrast visual acuity reduction associated with in vivo contact lens drying. Optom Vis Sci 1992;69:755-60.

16. Arrowsmith PN, Deitz MR, Marks RG, et al: Radial keratotomy: ARK study group. Thorofare NJ: Charles Slack, 1984.
17. Gartry DS, Kerr Muir MG, Marshall J. Excimer laser photorefractive keratectomy: 18 month follow-up. Ophthalmology 1992;99:1209-19.

18. Mellerio J, Palmer DA. Entoptic halos. Vision Res 1970; 10:595-9.

19. Munnerlyn CR, Koons JK, Marshall J. Photorefractive keratectomy: a technique for laser refractive surgery. J Cataract Refract Surg 1988;14:46-52. 